\title{
A new evaluation of the neutron data standards
}

\author{
A.D. Carlson ${ }^{1, a}$, V. Pronyaev ${ }^{2}$, G.M. Hale ${ }^{3}$, C. Zhenpeng ${ }^{4}$, R. Capote $^{5}$, I. Duran ${ }^{6}$, F.-J. Hambsch ${ }^{7}$, T. Kawano ${ }^{3}$, \\ S. Kunieda ${ }^{8}$, W. Mannhart ${ }^{9}$, B. Marcinkevicius ${ }^{5,14}$, R.O. Nelson ${ }^{3}$, D. Neudecker ${ }^{3}$, G. Noguere ${ }^{10}$, M. Paris $^{3}$,

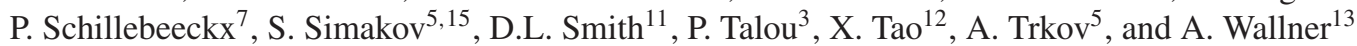 \\ 1 National Institute of Standards and Technology, 100 Bureau Dr. Stop 8463, Gaithersburg, MD 20899-8463, USA \\ 2 Atomsrandart, Rosatom State Corporation, Moscow, Russia \\ 3 Los Alamos National Laboratory, Los Alamos, NM 87545, USA \\ 4 Tsinghua University, Beijing 100084, China \\ 5 NAPC-Nuclear Data Section, International Atomic Energy Agency, Vienna, Austria \\ ${ }^{6}$ Facultad de Fisica, Universidad de Santiago de Compostela, Santiago de Compostela, La Coruña 15782, Spain \\ 7 EC-JRC-Dir. G, Unit G.2, Retieseweg 111, 2440 Geel, Belgium \\ ${ }^{8}$ Nuclear Data Center, Japan Atomic Energy Agency, 2-4 Shirane Shirakata, Tokai-mura, Naka-gun, Ibaraki 319-1195, Japan \\ 9 Neutron Metrology Group, Physikalisch-Technische Bundesanstalt, Org. 6.4, Bundesallee 100, 38116 Braunschweig, Germany \\ 10 SPRC/LEPh, CEA Cadarache, 13108 Saint Paul Les Durance, France \\ 11 Argonne Associate of Seville, Coronado, CA, USA \\ 12 China Nuclear Data Center (CNDC), China Institute of Atomic Energy, PO Box 275(41), Beijing 102413, China \\ 13 Research School of Physics and Engineering, The Australian National University, Nuclear Physics ACT 0200, Canberra, Australia \\ 14 Now at Uppsala University, Box 256, 75105 Uppsala, Sweden \\ 15 Now at Karlsruhe Institute of Technology, 76344 Eggenstein-Leopoldshafen, Germany
}

\begin{abstract}
Evaluations are being done for the $\mathrm{H}(\mathrm{n}, \mathrm{n}),{ }^{6} \mathrm{Li}(\mathrm{n}, \mathrm{t}),{ }^{10} \mathrm{~B}(\mathrm{n}, \alpha \gamma),{ }^{10} \mathrm{~B}(\mathrm{n}, \alpha), \mathrm{C}(\mathrm{n}, \mathrm{n}), \mathrm{Au}(\mathrm{n}, \gamma),{ }^{235} \mathrm{U}(\mathrm{n}, \mathrm{f})$ and ${ }^{238} \mathrm{U}(\mathrm{n}, \mathrm{f})$ standard cross sections. Evaluations are also being done for data that are not traditional standards including: the $\operatorname{Au}(\mathrm{n}, \gamma)$ cross section at energies below where it is considered a standard; reference cross sections for prompt gamma-ray production in fast neutron-induced reactions; reference cross sections for very high energy fission cross sections; the ${ }^{235} \mathrm{U}$ thermal neutron fission spectrum and the ${ }^{252} \mathrm{Cf}$ spontaneous fission neutron spectrum and the thermal constants.
\end{abstract}

\section{Introduction}

A number of national cross section data evaluation projects are planning new versions of their libraries including ENDF/B. Updated standards evaluations are needed for those libraries.

In response to requests for improvements in the standards, the Cross Section Evaluation Working Group (CSEWG), the Working Party on International Evaluation Cooperation (WPEC) of the Nuclear Energy Agency Nuclear Science Committee and the International Atomic Energy Agency (IAEA) are working cooperatively to provide new evaluations of the standards. Important contributions to the evaluation process from this joint international effort have been highlighted at several IAEA meetings.

\section{Evaluations}

The standards evaluation includes work on each of the following: the neutron cross section standards; the low energy gold capture cross section; reference cross sections for prompt gamma-ray production; very high energy fission reference cross sections; the ${ }^{235} \mathrm{U}$ thermal neutron-induced prompt fission neutron spectrum; the

a e-mail: carlson@nist.gov
${ }^{252} \mathrm{Cf}$ spontaneous prompt fission neutron spectrum and the thermal constants. The reference cross sections have the role of standards but they are not as well known. They have the same properties as the standards such as smooth cross sections as a function of energy. The work is still preliminary though it is nearly completed.

\subsection{The neutron cross section standards}

Cross section standards are needed for conversion of cross section measurements relative to those standards in order to produce new versions of cross section libraries. Improvements have been made in the very large database used for this standards evaluation. It includes the standards and ratios among them that can lead to improved evaluations of the standards. The cross sections being evaluated are $\mathrm{H}(\mathrm{n}, \mathrm{n}),{ }^{6} \mathrm{Li}(\mathrm{n}, \mathrm{t}),{ }^{10} \mathrm{~B}\left(\mathrm{n}, \alpha_{1} \gamma\right),{ }^{10} \mathrm{~B}(\mathrm{n}, \alpha)$, $\mathrm{C}(\mathrm{n}, \mathrm{n}), \mathrm{Au}(\mathrm{n}, \gamma),{ }^{235} \mathrm{U}(\mathrm{n}, \mathrm{f})$ and ${ }^{238} \mathrm{U}(\mathrm{n}, \mathrm{f})$. Also included in the evaluation process are the ${ }^{238} \mathrm{U}(\mathrm{n}, \gamma)$ and ${ }^{239} \mathrm{Pu}(\mathrm{n}, \mathrm{f})$ cross sections. Those data were included since there are many ratio measurements of those cross sections with the standards, and absolute data are available for them. The older measurements are given in [1] and the newer ones are shown in [2].

For details for the evaluation process for all the cross sections except those for the $\mathrm{H}(\mathrm{n}, \mathrm{n})$ and $\mathrm{C}(\mathrm{n}, \mathrm{n})$ reactions, see [1]. Basically the process involves using the GMAP

(c) The Authors, published by EDP Sciences. This is an Open Access article distributed under the terms of the Creative Commons Attribution License 4.0 (http://creativecommons.org/licenses/by/4.0/). 


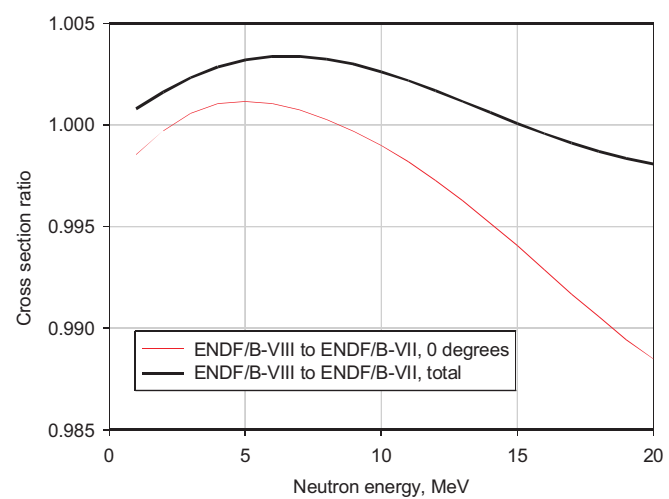

Figure 1. Comparison of the laboratory zero degree and total cross sections of hydrogen for the present evaluation with the previous standards evaluation.

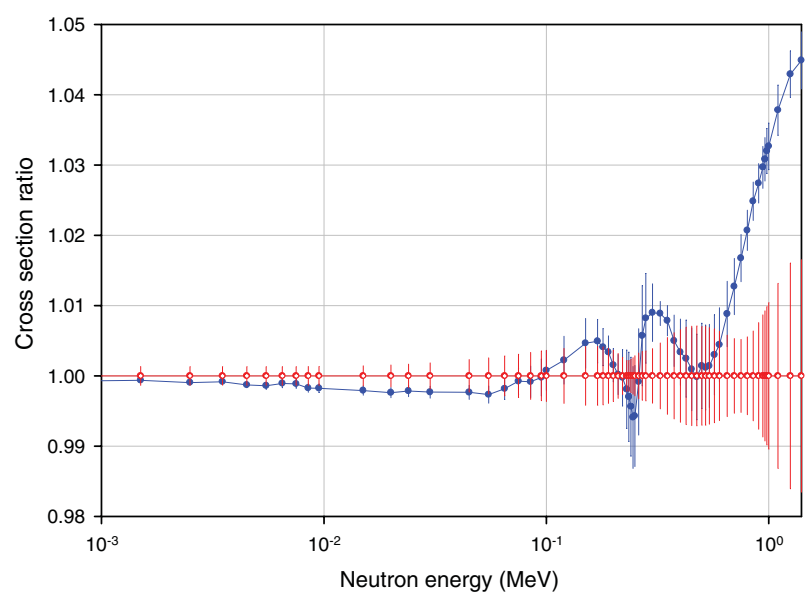

Figure 2. Ratio of the preliminary results of the present work to the previous standards evaluation for the ${ }^{6} \mathrm{Li}(\mathrm{n}, \mathrm{t})$ cross section.

(GMA) code to combine input from EDA and RAC R-matrix analyses; a thermal constants evaluation and direct experimental data to GMAP. The $H(n, n)$ and $C(n, n)$ evaluations are done separately as R-matrix analyses by Hale and Paris. The evaluation of the hydrogen standard is complete to $20 \mathrm{MeV}$. Efforts are underway to extend it to $200 \mathrm{MeV}$. The $\mathrm{C}(\mathrm{n}, \mathrm{n})$ evaluation, composed of separate ${ }^{12} \mathrm{C}(\mathrm{n}, \mathrm{n})$ and ${ }^{13} \mathrm{C}(\mathrm{n}, \mathrm{n}) \mathrm{R}$-matrix evaluations, is given in a paper [3] at this conference. The energy ranges of the standards can be found in [1]. In Figs. 1-7 comparisons are made of the results of the new evaluation with those of the previous evaluation. For many of the cross sections, only small changes occurred due to the consistency of the new data with the previous standards.

For the ${ }^{6} \mathrm{Li}(\mathrm{n}, \mathrm{t})$ cross section, there were differences in the fits for the EDA and RAC R-Matrix analyses, so the simple averages of the two fits with increased uncertainties were used in the combined GMA fit with the other data. An additional component of uncertainty equal to the difference between the two fits was used in the GMAP analysis. Large uncertainty is observed in the region of the resonance at about $0.245 \mathrm{MeV}$. The increase above $0.5 \mathrm{MeV}$ was present in both fits. The data above $20 \mathrm{MeV}$ will have some changes when the new hydrogen evaluation is completed.

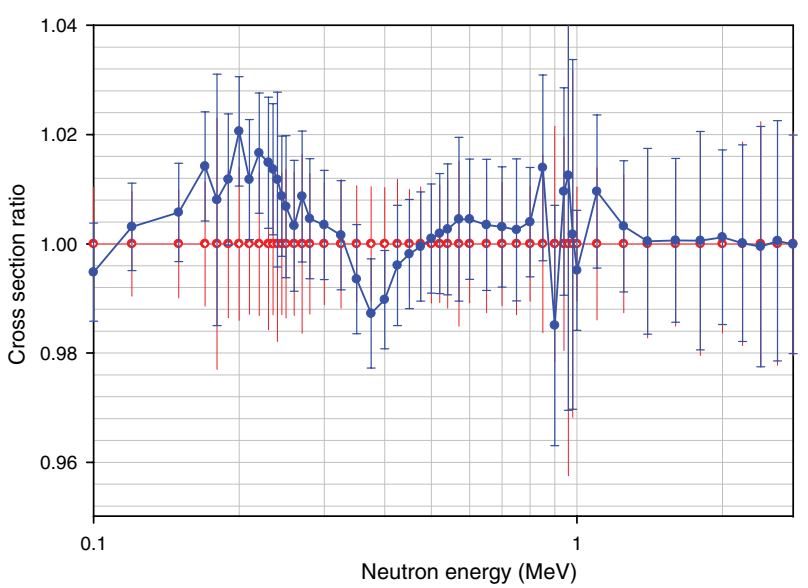

Figure 3. Ratio of the present evaluation of the $\mathrm{Au}(\mathrm{n}, \gamma)$ cross section with the previous standards evaluation.

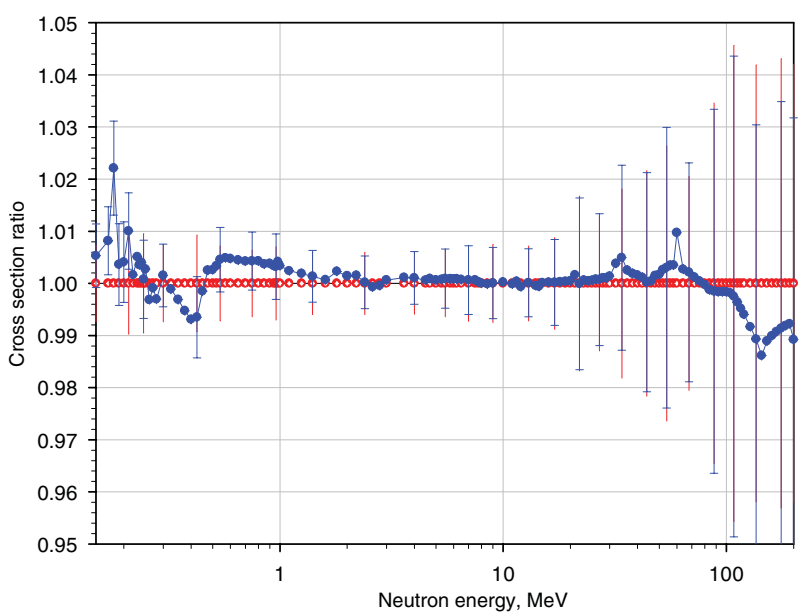

Figure 4. Comparison of the ${ }^{235} \mathrm{U}(\mathrm{n}, \mathrm{f})$ cross section from this evaluation with the previous standards evaluation.

\subsection{Thermal constants}

These data are particularly important in the determination of the neutron economy in thermal reactors. Since thermal data are included in the standards evaluation, the thermal constants will have an impact on the results of the evaluation. Also the thermal constants themselves will be affected by the other data in the evaluation.

Due to concerns about how well the temperatures were understood for Maxwellian data, only microscopic data were used in the evaluation as input in the GMAP code. Thus the Westcott g-factors for fission and absorption normally given with the thermal constants were not evaluated. The results of this work will be given in a paper [4] ] at this conference.

\subsection{Low energy $\mathrm{Au}(\mathrm{n}, \gamma)$ cross section}

The Maxwellian averaged cross section at $\mathrm{kT}=30 \mathrm{keV}$ for ${ }^{197} \mathrm{Au}(\mathrm{n}, \gamma)$ is used in neutron capture cross-section measurements as a reference for reactions important for astrophysics. This reference cross section was obtained from an evaluation based on the results of measurements by Ratynski and Käppeler [5] of the ${ }^{197} \mathrm{Au}(\mathrm{n}, \gamma)$ cross section averaged over a Maxwellian-like experimentally simulated spectrum with temperature near $30 \mathrm{keV}$ as well as from measurements by Macklin [6]. The present 


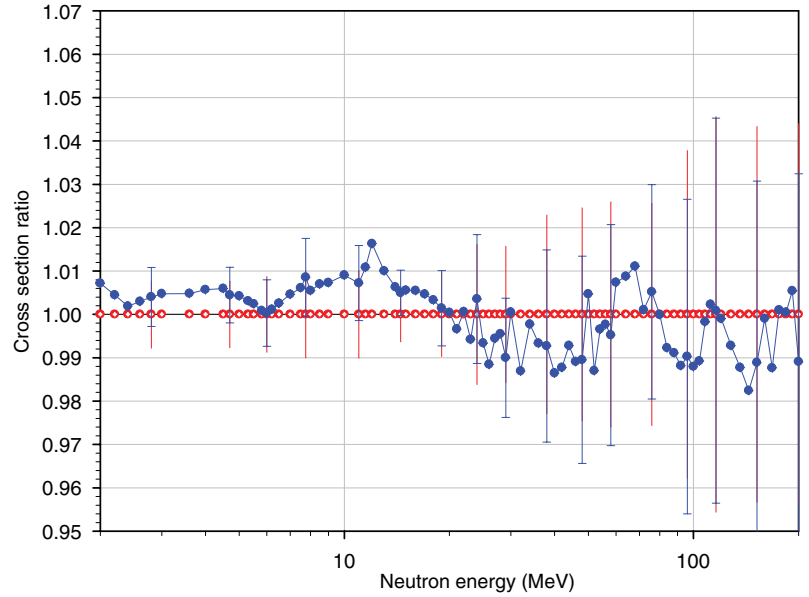

Figure 5. Comparison of the ${ }^{238} \mathrm{U}(\mathrm{n}, \mathrm{f})$ cross section from this evaluation with the previous standards evaluation.

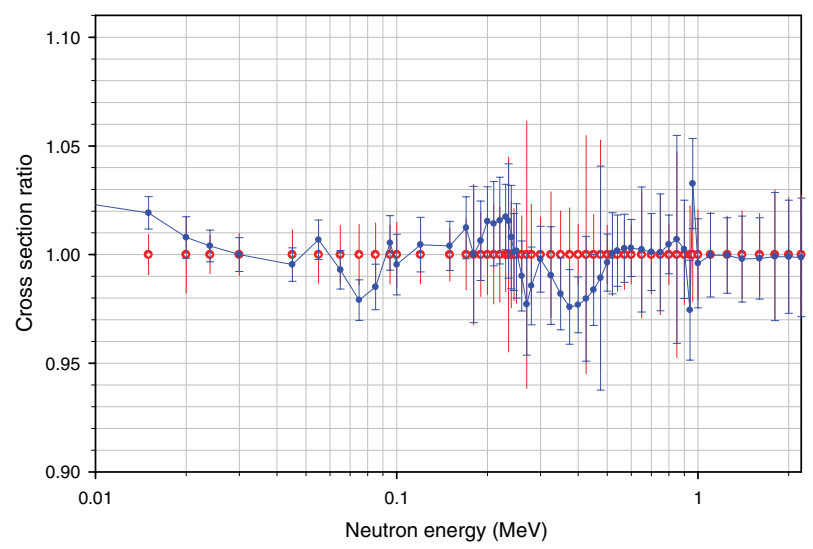

Figure 6. Ratio of the ${ }^{238} \mathrm{U}(\mathrm{n}, \gamma)$ cross section from this evaluation to the previous standards evaluation.

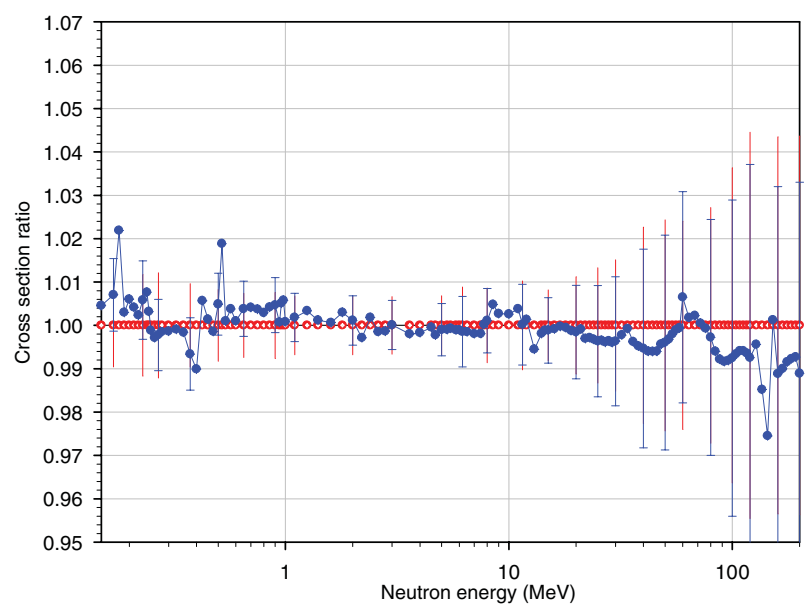

Figure 7. Ratio of the ${ }^{239} \mathrm{Pu}(\mathrm{n}, \mathrm{f})$ cross section from this evaluation to the previous standards evaluation.

evaluation is approximately $5 \%$ to $7 \%$ above the Ratynski and Käppeler evaluation. Because of this discrepancy several new experiments and re-analyses were done. The results support the present evaluation. In Fig. 8, results of recent experiments $[7,8]$ ] are compared with the previous and present standards evaluation.

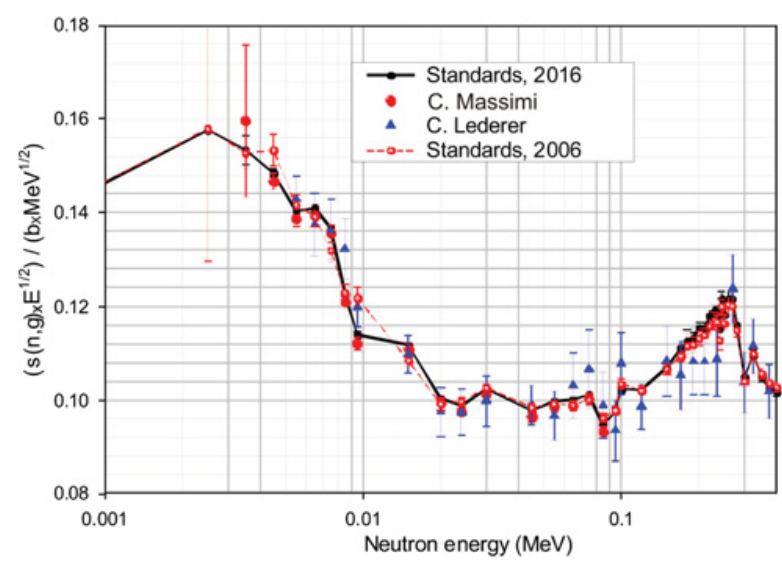

Figure 8. Comparison of recent measurements and evaluations of the ${ }^{197} \mathrm{Au}(\mathrm{n}, \gamma)$ cross section in the $30 \mathrm{keV}$ energy region.

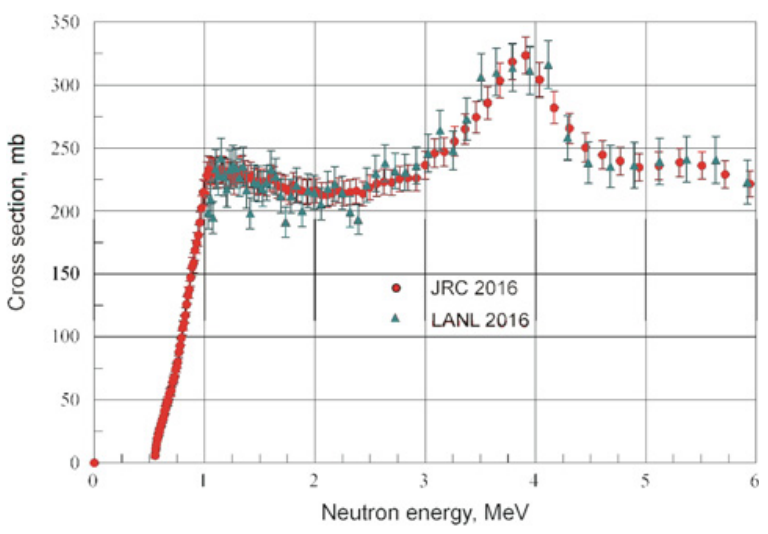

Figure 9. Recent measurements of the ${ }^{7} \operatorname{Li}\left(\mathrm{n}, \mathrm{n}^{\prime} \gamma\right)$ cross section.

\section{4. $\gamma$-ray production reference cross sections}

Measurements of neutron-induced $\gamma$-ray production cross sections are most easily performed using a reference cross section in which a discrete $\gamma$-ray is detected. Reference cross sections for such measurements have been evaluated. The reference cross sections are the ${ }^{10} \mathrm{~B}(\mathrm{n}, \alpha \gamma)$ cross section up to $1 \mathrm{MeV}\left(\mathrm{E}_{\gamma}=0.478 \mathrm{MeV}\right)$; the ${ }^{7} \mathrm{Li}\left(\mathrm{n}, \mathrm{n}^{\prime} \gamma\right)$ cross section from $0.8 \mathrm{MeV}-5 \mathrm{MeV}\left(\mathrm{E}_{\gamma}=0.478 \mathrm{MeV}\right)$ and the ${ }^{48} \mathrm{Ti}\left(\mathrm{n}, \mathrm{n}^{\prime} \gamma\right)$ cross section from 3 to $15 \mathrm{MeV}$ $\left(\mathrm{E}_{\gamma}=0.984 \mathrm{MeV}\right)$. The recent JRC [9] and LANL [10] measurements of the ${ }^{7} \operatorname{Li}\left(n, n^{\prime} \gamma\right)$ cross section shown in Fig. 9 are in good agreement and suggest that the evaluation of these data will provide a good reference cross section. Recent measurements at JRC [11] and LANL [12] of the ${ }^{48} \operatorname{Ti}\left(n, n^{\prime} \gamma\right)$ cross section are shown in Fig. 10 compared with the GMAP evaluation [13] which is the reference cross section.

\subsection{High energy reference fission cross section}

Reference cross sections at high energies are needed for conversion of ratio measurements to cross sections at those energies.

GMAP evaluations [14] have been made of the ${ }^{209} \mathrm{Bi}(\mathrm{n}, \mathrm{f})$ and ${ }^{\text {nat }} \mathrm{Pb}(\mathrm{n}, \mathrm{f})$ cross sections up to $1 \mathrm{GeV}$ as reference cross sections. As part of that work the ${ }^{235} \mathrm{U}(\mathrm{n}, \mathrm{f})$, ${ }^{238} \mathrm{U}(\mathrm{n}, \mathrm{f})$ and ${ }^{239} \mathrm{Pu}(\mathrm{n}, \mathrm{f})$ cross sections have been extended up to $1 \mathrm{GeV}$. The ${ }^{235} \mathrm{U}(\mathrm{n}, \mathrm{f})$ and ${ }^{238} \mathrm{U}(\mathrm{n}, \mathrm{f})$ cross sections are reference cross sections, not standards from $200 \mathrm{MeV}$ to $1 \mathrm{GeV}$. These evaluations rely strongly on $(\mathrm{p}, \mathrm{f})$ cross 


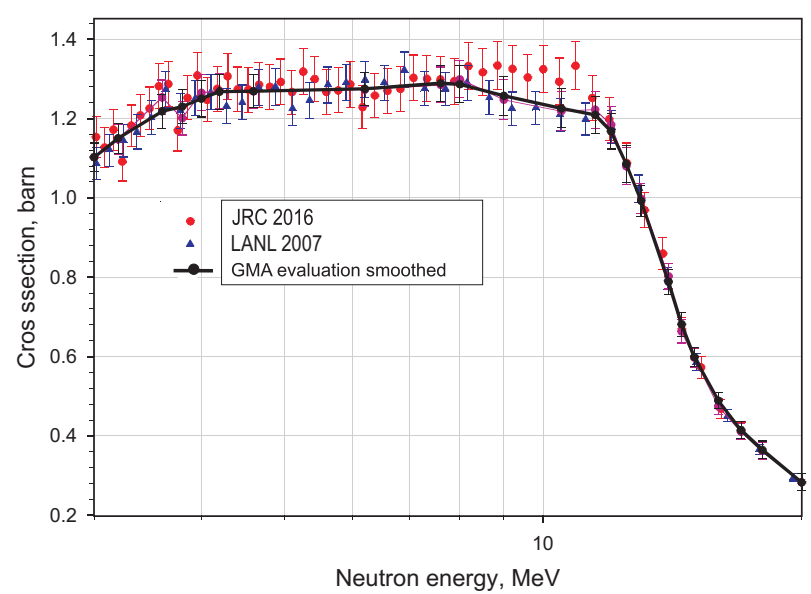

Figure 10. Recent measurements of the ${ }^{48} \operatorname{Ti}\left(\mathrm{n}, \mathrm{n}^{\prime} \gamma\right)$ cross section $(\mathrm{E}=0.984 \mathrm{MeV})$ compared with the GMA evaluation.

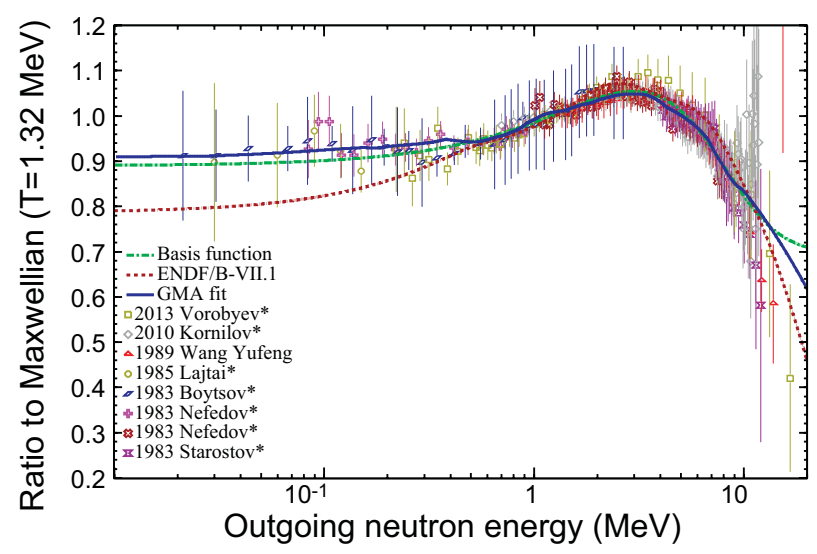

Figure 11. ${ }^{235} \mathrm{U}$ thermal prompt neutron fission spectrum evaluations and measurements.

sections at very high energies since very little $(\mathrm{n}, \mathrm{f})$ data are available. At the highest energies the $(n, f)$ and $(p, f)$ cross section differences are small compared with evaluated uncertainties.

\subsection{Prompt fission spectra}

The ${ }^{235} \mathrm{U}$ thermal prompt neutron fission spectrum (PFNP) is very important for reactor applications. It is also used as a reference for validating evaluated cross sections for neutron dosimeters used in many applications. Improvements in the evaluation of this spectrum, were made by including measurements of the spectrum made relative to the ${ }^{252} \mathrm{Cf}$ spontaneous fission neutron spectrum. The evaluation was done with GMAP for which both spectra were evaluated simultaneously, and by considering all ratio measurements as shape data [15].

Due to the much smaller uncertainties of the ${ }^{252} \mathrm{Cf}$ spectrum, the impact was largely on the ${ }^{235} \mathrm{U}$ spectrum. The ${ }^{252} \mathrm{Cf}$ spontaneous fission neutron spectrum evaluation of Mannhart [1] was used in this evaluation. The results of this work were extensively documented in [16] and will be given in a paper [17] at this conference. The average energy of this spectrum is $2.00 \mathrm{MeV}$ with an uncertainty of $0.01 \mathrm{MeV}$. In Fig. 11 preliminary results for the thermal PFNS for ${ }^{235} \mathrm{U}$ compared with measurements [18-24] are shown. The preliminary evaluation is the GMA fit up to $10 \mathrm{MeV}$ and the basis function at higher energies.

\section{Conclusions}

This international effort is leading to evaluations needed for new versions of cross section libraries. Most changes in data are relatively small. A comprehensive report on the evaluation process is being prepared for publication in Nuclear Data Sheets.

\section{References}

[1] A.D. Carlson, et al., Nucl. Data Sheets 100, 3215 (2009)

[2] A.D. Carlson, et al., EPJ Web of Conferences 106, 04002 (2016)

[3] G.M. Hale and M. Paris, contribution to this conference

[4] V.G. Pronyaev, et al., contribution to this conference

[5] W. Ratynski and F. Käppeler, Phys. Rev. C37, 595 (1988)

[6] R.L. Macklin, Nucl. Sci. Eng. 79, 265 (1981)

[7] C. Lederer, et al., Phys. Rev. C83, 034608 (2011)

[8] C. Massimi, et al., EPJA 50, 124 (2014)

[9] N. Nyman, et al., Phys. Rev. C93, 024610 (2016)

[10] R. Nelson, Private communication (2016)

[11] A. Olacel, et al., JRC Technical Report EUR 27621 EN (2015) and Private communication (2016)

[12] D. Dashdorj, et al., Nucl. Sci. Eng. 157, 65 (2007)

[13] S.P. Simakov, et al., CERN-Proceedings-2012002325 (2012) and private communication from V. Pronyaev (2016)

[14] B. Marcinkevicius, S. Simakov and V. Pronyaev, INDC(NDS)-0681(2015)

[15] D.L. Smith, D. Neudecker, R. Capote Noy, INDC(NDS)-0678 (2015)

[16] R. Capote, et al., Nucl. Data Sheets 131, 1 (2016)

[17] R. Capote, et al., contribution to this conference

[18] A.S. Vorobyeva, et al., EPJ Web Conf 8, 03004 (2010)

[19] N. Kornilov, et al., Nucl. Sci. Eng. 165, 117 (2010)

[20] Y. Wang, et al., CNP 11, 47 (1989)

[21] A. Lajtai, et al., Proceedings of the Nuclear Data for Basic and Applied Science Conf., Santa Fe, p. 613 (1985)

[22] B.I. Starostov, et al., 6th All Union Conf. Kiev 2, 290 (1983)

[23] A.A. Bojcov, et al., ibid. 2, 294 (1983)

[24] V.N. Nevedov, et al., ibid. 2, 285 (1983) 